04,05

\title{
Structure and Magnetodielectric Properties of Composite Ceramics Based on Lead Ferroniobate
}

\author{
(C) A.A. Fedii ${ }^{1}$, D.A. Kalganov ${ }^{2}$, E.M. Filonenko ${ }^{1}$, F.A. Yaroshenko ${ }^{1}$, \\ P.A. Bezborodova ${ }^{1}$, Yu.A. Lupitskaya ${ }^{1, \uparrow}$ \\ ${ }^{1}$ Chelyabinsk State University, \\ Chelyabinsk, Russia \\ 2 ITMO University, \\ St. Petersburg, Russia \\ ๆ E-mail: lupitskaya@gmail.com
}

Received: July 8, 2021

Revised: July 13, 2021

Accepted: July 16, 2021

In this research, the structural, electrodynamic, and magnetodielectric properties of composite ceramic materials based on lead ferroniobate $\mathrm{Pb}\left(\mathrm{Fe}_{0.5} \mathrm{Nb}_{0.5}\right) \mathrm{O}_{3}$ have been studied. The experimentally obtained temperature and frequency dependences of the dielectric constant indicate the presence of a low-temperature polar state, large values of the dielectric constant in the microwave range, and high electrical conductivity of the materials studied.

Keywords: lead ferroniobate, composite materials, ferroelectric phases, ceramics, magnetodielectric properties.

DOI: 10.21883/FTT.2021.11.51575.14s

\section{Introduction}

Materials with simultaneous magnetic and electrical ordering (multiferroics) are promising for use in modern microelectronics and microwave electronics [1]. The connection between the magnetic and electrical subsystem allows controlling the corresponding properties of these materials using external electric and magnetic fields. The number of compounds exhibiting the properties of multiferroics at temperatures close to room temperature is considered to be insufficient since in most cases electrical ordering at high temperatures is incompatible with magnetic [2].

Composites based on lead ferroniobate $\mathrm{Pb}\left(\mathrm{Fe}_{0.5} \mathrm{Nb}_{0.5}\right) \mathrm{O}_{3}$ (PFN) belong to the widely known and best studied multiferroics with phase transition temperatures corresponding to electric and magnetic ordering $T_{\mathrm{C}}=(356 \pm 5) \mathrm{K}$ and $T_{\mathrm{N}}=(150 \pm 10) \mathrm{K}$. Due to the equal content of triand pentavalent ions in PFN, a stable structure of the $\mathrm{ABO}_{3}$ perovskite type is formed with anti-ferromagnetic and ferroelectric properties, for which $\mathrm{BO}_{6}$ octahedrons containing, respectively, $\mathrm{Fe}^{3+}$ and $\mathrm{Nb}^{5+}$ are responsible.

At room temperature, PFN possesses only ferroelectric properties [3]; however, high polarization values in this material are of practical interest when creating new materials for microwave converters and composite magnetoelectric materials $[2,4]$.

In composite structures, magnetoelectric (ME) effects arise as a result of a combination of magnetostriction in the magnetic phase and the piezoelectric effect in the ferroelectric phase. In this case, the efficiency of ME interactions in composite structures can be increased by selecting magnetic materials with a large piezomagnetic coefficient and high saturation magnetization, as well as electrical materials with a high piezomodule and low dielectric losses. Thus, it is necessary to study the electrodynamic characteristics of PFN ceramics in the microwave range, as well as the development and study of new composite magnetoelectric materials based on it.

The presented work is dedicated to studying the temperature dependence of the dielectric constant and the tangent of the loss angle for ceramic samples of PFN and a composite material based on it in the high-frequency and microwave ranges.

\section{Experimental}

All samples were obtained by the solid-phase reaction method using high-purity starting components (laboratory reagent grade/reagent grade). The oxides $\mathrm{Fe}_{2} \mathrm{O}_{3}$ and $\mathrm{Nb}_{2} \mathrm{O}_{5}$ were preliminarily thermally treated at a temperature of $1273 \mathrm{~K}$. In order to obtain polycrystalline PFN samples, two different methods were used: two-stage (using the $B$-precursor $\mathrm{FeNbO}_{4}$, described in detail in [3]) and direct synthesis from the starting components.

During the direct synthesis, the starting components were mixed according to the reaction equation:

$$
4 \mathrm{PbO}+\mathrm{Fe}_{2} \mathrm{O}_{3}+\mathrm{Nb}_{2} \mathrm{O}_{5} \rightarrow 4 \mathrm{~Pb}\left(\mathrm{Fe}_{0.5} \mathrm{Nb}_{0.5}\right) \mathrm{O}_{3} .
$$

To prevent the formation of pyrochlore phases and reduce the conductivity of the PFN ceramics, a 1\%-mass fraction of lithium carbonate $\mathrm{Li}_{2} \mathrm{CO}_{3}$ was introduced into the resulting mixture. The resulting mixture then was used to form cylindrical specimens with a diameter of $14 \mathrm{~mm}$ and a thickness of $1-2 \mathrm{~mm}$ at a pressure of $100 \mathrm{MPa}$ and temperature of $1123 \mathrm{~K}$ for $4 \mathrm{~h}$. 
In order to obtain a composite magnetoelectric material, copper ferromanganite $\mathrm{CuMnFeO}_{4}(\mathrm{CMF})$ synthesized by the technology described in detail in [5], as well as PFN samples obtained by a two-stage method were used. The starting compounds were mixed in equal weight proportions $0.5 \mathrm{PFN}+0.5 \mathrm{CMF}$ in an agate mortar for $30 \mathrm{~min}$., then pressed at a pressure of $200 \mathrm{MPa}$, and sintered at a temperature of $673 \mathrm{~K}$ during the time of $2 \mathrm{~h}$.

The phase composition control and the study of the obtained samples crystal structure were conducted by electron microscopy and $X$-ray structural analysis methods. $X$-ray diffraction patterns were obtained with the help of DRON-3 diffractometer $\left(\mathrm{Cu} K_{\alpha}\right.$ radiation $)$ at room temperature with an angle step of $0.01^{\circ}$ in the angle range $10 \leq 2 \Theta \leq 80^{\circ}$. To obtain electron microscopic images and study the elemental composition of compounds, the JEOL JSM-6510 scanning electron microscope at an accelerating voltage of $15.0 \mathrm{kV}$ without preliminary sample preparation was applied.

In order to analyze the temperature dependences of the PFN dielectric constant $\varepsilon$ in the frequency range from 20 to $300 \mathrm{kHz}$, thin (up to $1 \mathrm{~mm}$ ) cylindrical samples were placed in a measuring flat-capacitor type cell with silver clamping electrodes of $14 \mathrm{~mm}$ diameter.

The value of the capacitor with the test sample and the loss tangent were measured using an Aktakom AM-3028 RLC analyzer. The dielectric constant was calculated using the formula of the flat capacitor.

The electrodynamic characteristics of the samples in the centimeter range of electromagnetic waves were investigated by the resonance method at a frequency of $9.163 \mathrm{GHz}$. The values of the permittivity and magnetic permeability were obtained from the shift of the resonant frequency and the change in the $Q$-factor of a rectangular resonator [6]. In the microwave frequency range of $8-40 \mathrm{GHz}$, the transmission and reflection coefficients were measured using the method of partial filling of a rectangular waveguide [6].

\section{Results and discussion}

Phase composition $X$-ray analysis was applied for all stages of samples synthesis. Fig. 1 shows the $X$-ray diffraction patterns of the precursor and two synthesized PFN samples. The $X$-ray diffraction pattern of the $\mathrm{FeNbO}_{4}$ precursor (Fig. 1, $a$ ) is in good agreement with the known data $[3,7]$.

Referring to the study of PFN and CMF samples diffraction patterns, it indicates a high degree of obtained compounds crystallinity and their single-phase composition (Fig. 2, $a$ and $b$ ). Ferromanganite CMF corresponds to a cubic crystal structure (space group $F d-3 m$ ) with unit cell parameters $a=b=c=8.40 \AA$. The data of qualitative $X$-ray phase analysis showed that the initial phases (PFN and CMF, respectively) form the synthesized composite without changing the symmetry of the samples crystal lattice, and the intensity of the diffraction maxima corresponds to the volume fraction of the heterogeneous

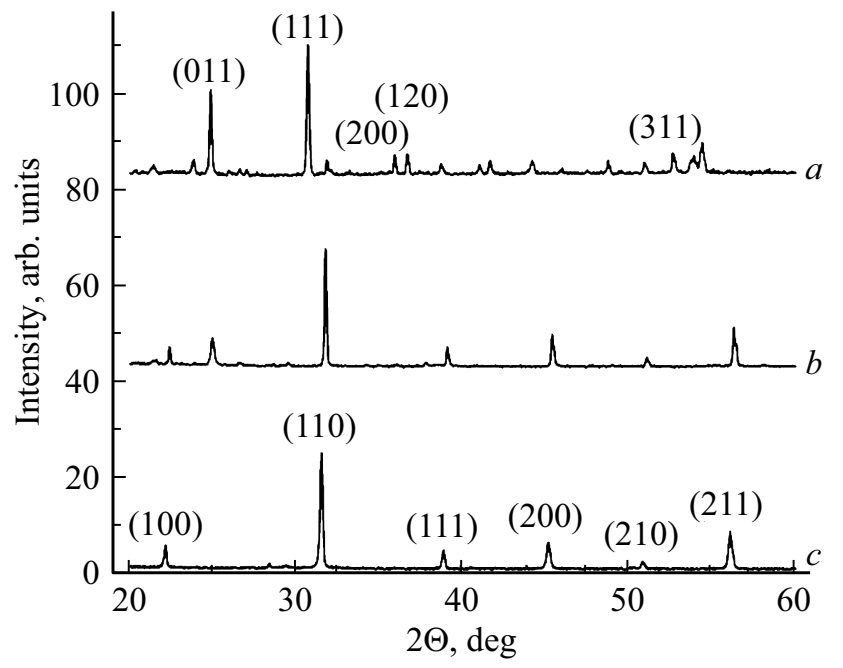

Figure 1. $X$-ray diffraction patterns of $a$ ) the precursor $\mathrm{FeNbO}_{4}$, and PFN ceramics synthesized by $b$ ) one- and $c$ ) two-stage methods.

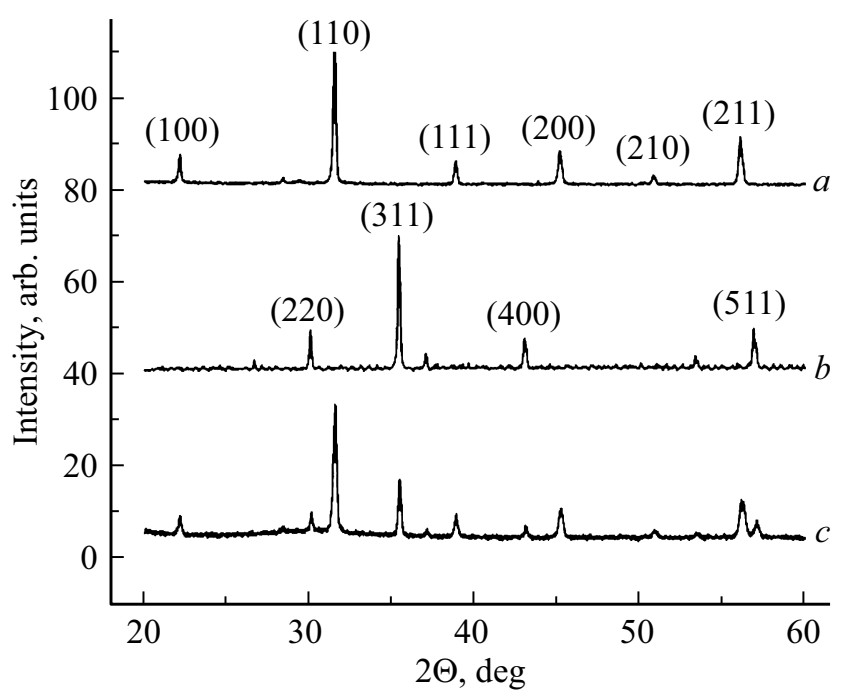

Figure 2. $X$-ray diffraction patterns of samples: $a$ ) PFN, $b$ ) ferromanganite $\mathrm{CMF}$, and $c$ ) composite material $0.5 \mathrm{PFN}+0.5 \mathrm{CMF}$.

system components (Fig. 2,c). Thus, the $X$-ray diffraction pattern of the composite material with the composition $0.5 \mathrm{PFN}+0.5 \mathrm{CMF}$ (Fig. 2,c) at diffraction angles of 22, 32, $39,45,51$, and $57^{\circ}$ shows a strictly defined sequence of maxima related to the compound of the PFN composition (Fig. 2,a). At the same time, the $X$-ray diffraction pattern of the $0.5 \mathrm{PFN}+0.5 \mathrm{CMF}$ composite (Fig. 2,c) at diffraction angles of $30,36,43,54$, and $57^{\circ}$ shows a strict set of maxima belonging to the CMF sample (Fig. 2,b). It should be also noted that the $X$-ray diffraction patterns of the synthesized initial components PFN and CMF for the composite material $0.5 \mathrm{PFN}+0.5 \mathrm{CMF}$ do not contain impurity phases.

Further comprehensive study included obtaining electron microscopic images of PFN ceramics and composite ma- 


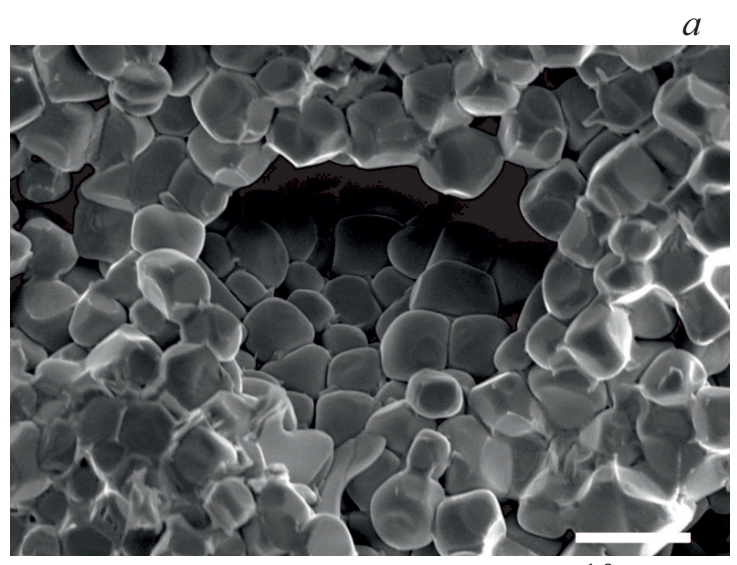

$10 \mu \mathrm{m}$

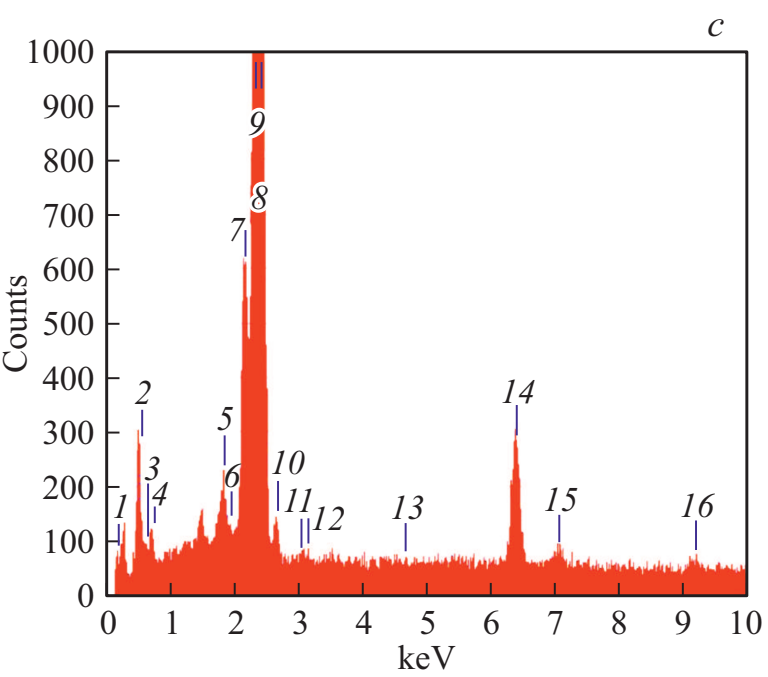

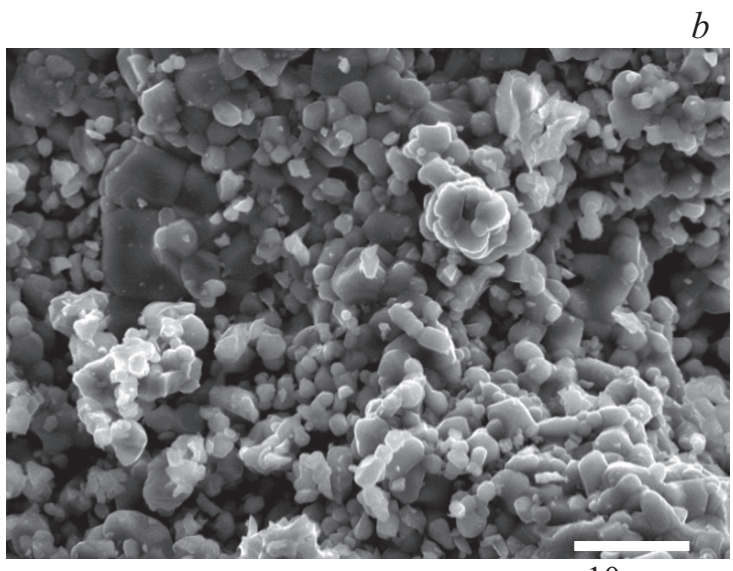

$10 \mu \mathrm{m}$

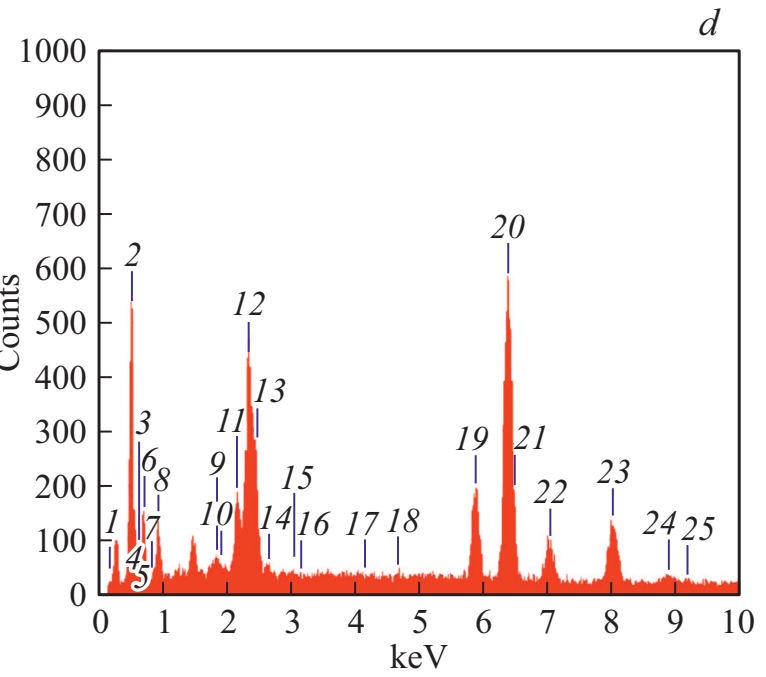

Figure 3. Electron microscopic images of the crystal structure and elemental analysis spectra of PFN $(a, c)$ and composite material $0.5 \mathrm{PFN}+0.5 \mathrm{CMF}(b, d)$, respectively. For $c$-diagram: $1-\mathrm{NbMz} ; 2-\mathrm{OKa} ; 3-\mathrm{FeLl} ; 4-\mathrm{FeLa} ; 5-\mathrm{PbMz} ; 6-\mathrm{NbLl} ; 7-\mathrm{NbLa}$ PbM3-N; $8-$ PbMa; $9-$ PbMb; $10-$ PbMr; $11-$ PbM3-O; $12-$ PbM2-N; $13-$ FeKesc; $14-$ FeKa; $15-$ FeKb; $16-\mathrm{PbL} 1$. For $d$-diagram: $1-\mathrm{NbMz} ; 2-\mathrm{OKa} ; 3-\mathrm{MnLl} ; 4-\mathrm{FeLl} ; 5-\mathrm{MnLa} ; 6-\mathrm{FeLa} ; 7-\mathrm{CuLl} ; 8-\mathrm{CuLa} ; 9-\mathrm{PbMz} ; 10-\mathrm{NbLl}$; 11 - NbLa PbM3-N; 12 - PbMa; 13 - PbMb; 14 - PbMr; 15 - PbM3-O; 16 - PbM2-N; 17 - MnKesc; $18-$ FeKesc; $19-\mathrm{MnKa} ; 20-\mathrm{FeKa} ; 21-\mathrm{MnKb} ; 22-\mathrm{FeKb} ; 23-\mathrm{CuKa} ; 24-\mathrm{CuKb} ; 25-\mathrm{PbL}$.
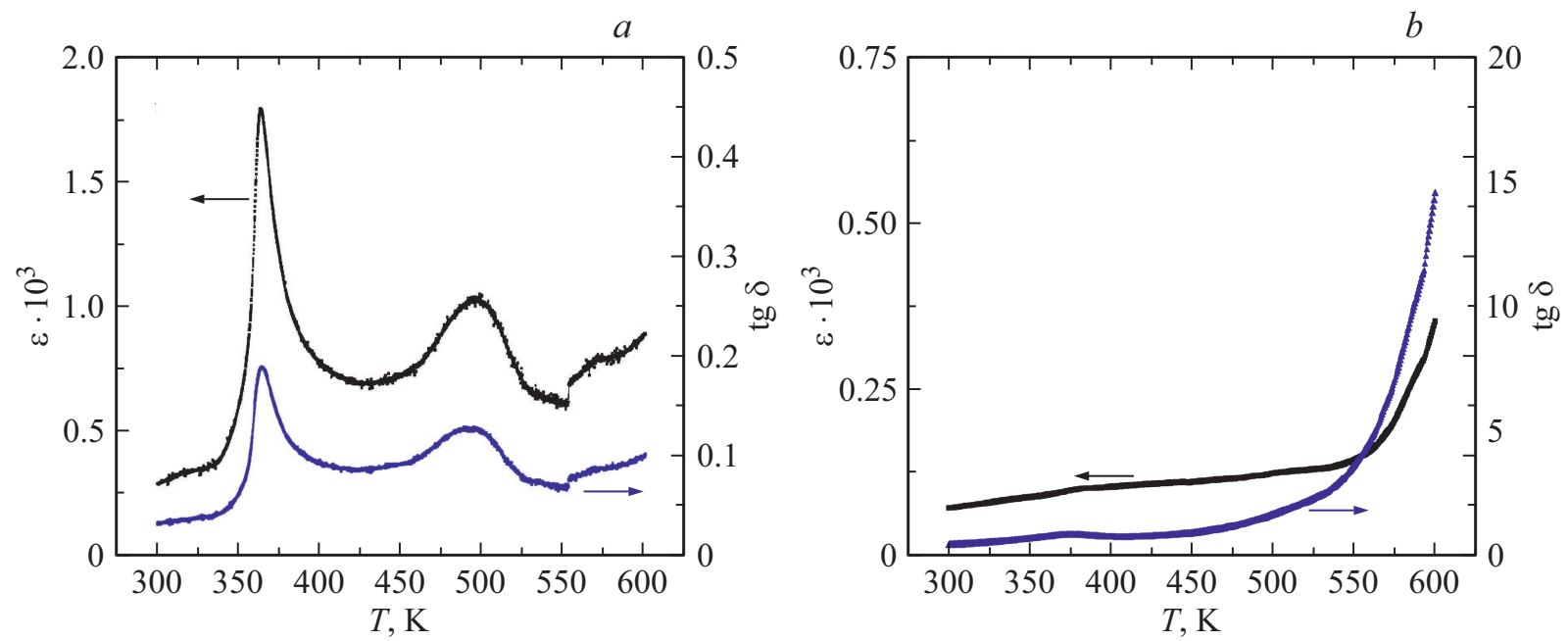

Figure 4. Temperature dependence of dielectric constant and loss tangent for $a$ ) PFN sample and $b$ ) composite material $0.5 \mathrm{PFN}+0.5 \mathrm{CMF}$ at $10 \mathrm{kHz}$. 

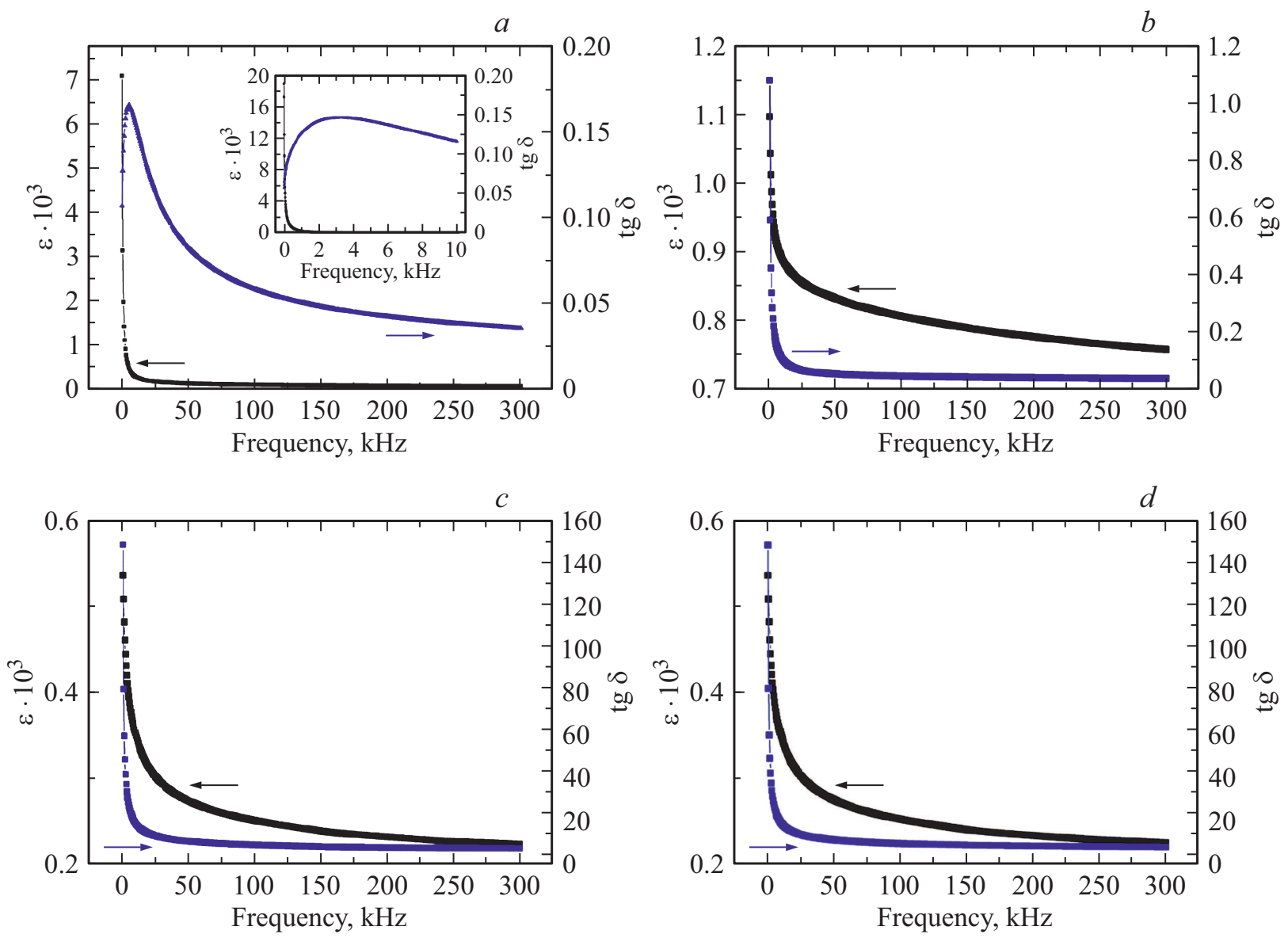

Figure 5. Frequency dependence of permittivity $\varepsilon$ and loss tangent $\tan \delta$ at temperature $T_{\mathrm{l}}=300 \mathrm{~K}$ and $T_{\mathrm{h}}=600 \mathrm{~K}$, for samples $(a, b)$ PFN and composite material $(c, d)$, respectively.

terial samples with a magnification of $\times 2000$ (Fig. 3, $a$ and $b)$ as well an elemental analysis of the obtained samples conduction. According to electron microscopy data, the crystallite size of PFN ceramics is $5-8 \mu \mathrm{m}$. In the composite material, CMF particles with a size of $1-3 \mu \mathrm{m}$ are evenly located between crystallites of lead ferroniobate. Elemental analysis data (Fig. 3, $c$ and $d$ ) confirm the mass fractions of elements for the stoichiometric composition of PFN and CMF compounds and composite material with a molar ratio of 0.5 .

The temperature dependence of the dielectric constant and the loss tangent (Fig. 4,a) was used to determine the temperature of lead ferroniobate transition from the ferroelectric to the paraelectric phase. i. e., the Curie point $\left(T_{c} \approx 370 \mathrm{~K}\right)$. At this temperature, a pronounced maximum of $\varepsilon$ is observed.

The maximum permittivity at $490 \mathrm{~K}$ can be explained by the $R 3 m-C m-P m-3 m$ structural transition described in detail in [8]. In the samples of the composite material, the maximum $\varepsilon(T)$ corresponding to the Curie point PFN is blurred due to large losses (Fig. 4, $b$ ). As the temperature rises above $T=550 \mathrm{~K}$, the increase in the dielectric constant and losses due to thermal polarization is observed.
The frequency dependences of $\varepsilon^{\prime}$ and $\tan \delta$ for the samples under study at a temperature ${ }_{1}=300 \mathrm{~K}<T_{\mathrm{C}}$ and $T_{\mathrm{h}}=600 \mathrm{~K}>T_{\mathrm{C}}$ are shown in Fig. 5. The measurements were carried out in the range of $0.5-300 \mathrm{kHz}$ with a frequency step of $500 \mathrm{~Hz}$.

The dispersion of the permittivity corresponds to the polar state of the lead ferroniobate crystals at a temperature below the ferroelectric-paraelectric phase transition (Fig. 5, $a$ ). At a frequency of $f \approx 1.5 \mathrm{kHz}$, a relaxation maximum of dielectric losses is observed. The absence of the $\varepsilon^{\prime}$ maximum at the same frequencies is explained by the high electrical conductivity of the PFN. No relaxation maxima are observed at $T_{\mathrm{h}}=600 \mathrm{~K}>T_{\mathrm{C}}$.

The magnetodielectric effect (MDE) at a temperature of $T=300 \mathrm{~K}$ in a composite material was measured according to the planar capacitor scheme in a transverse external magnetic field $\mu_{0} H=0.2 \mathrm{~T}$ :

$$
\mathrm{MD}=\frac{\varepsilon_{H}^{\prime}-\varepsilon_{0}^{\prime}}{\varepsilon_{0}^{\prime}} \cdot 100 \%, \quad \mathrm{ML}=\frac{\operatorname{tg} \delta_{H}-\operatorname{tg} \delta_{0}}{\operatorname{tg} \delta_{0}} \cdot 100 \%
$$

where $\varepsilon_{H}^{\prime}$ and $\operatorname{tg} \delta_{H}$ are the values of the dielectric constant and the tangent of the loss angle in an external magnetic field, and $\varepsilon_{0}$ and $\operatorname{tg} \delta_{0}$ - without magnetic field (Fig. 6). 


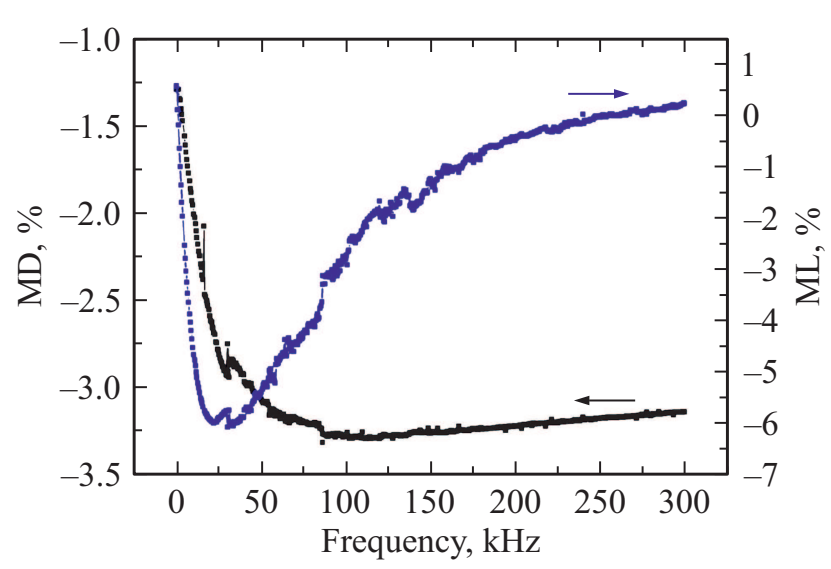

Figure 6. The frequency dependence of the MDE for the real (MD) and imaginary (ML) parts of the permittivity.

Studies of the synthesized samples in $(20-300) \mathrm{Hz}$ frequency range at various temperatures allow determining the temperature of the second-order phase transition. Thus, the temperature dependence of the dielectric constant and the tangent of the dielectric loss angle (Fig. 4,a) was used to determine the transition temperature (ferroelectric-paraelectric) of the PFN sample. However, the experimentally obtained data follow that at $f \sim 25 \mathrm{kHz}$, a minimum of the magnetodielectric coefficient is observed and disappears, with a further increase in frequency, caused by an increase in electrical conductivity in the composite material (an increase in dielectric losses in a given frequency region, Fig. 5). High values of electrical conductivity in the test material mask the maximum dielectric constant corresponding to the ferroelectric and phase transition. The authors of this article suggest that the observed decrease in the magnetodielectric effect with increasing frequency is not associated with resonance phenomena.

PFN ceramics is characterized by a fairly uniform grain structure (Fig. 3,a), consisting of crystallites in the form of polyhedrons with an average size of $\sim 5-8 \mu \mathrm{m}$. In the study of a thin section of the object surface by the SEM method, the presence of inclusions of an impurity phase $(\sim 0.3$ vol.\% $) \mathrm{Fe}_{2} \mathrm{O}_{3}$ was revealed. Apparently, the presence of impurities in the sample is the reason for the appearance of spontaneous magnetization at room temperature. The most probable model for ceramics based on PFN seems to be a model, in which the magnetoelectric effect is associated with a shift in the magnetic field of the ferro-paraelectric phase transition temperature [9].

Since the values of the dielectric constant and losses in the materials under study strongly depend on the frequency, it is necessary to analyze their electrodynamic characteristics in the microwave range of electromagnetic waves, which will allow drawing a conclusion about the possible practical application of such materials.

The shift of the resonance frequency and the change in the $Q$-factor of the resonator during the study of the samples electrodynamic characteristics in the centimetre range of electromagnetic waves are shown in Fig. 7.

The resonance frequencies and $Q$-factors of a rectangular resonator with a sample of small dimensions $(1 \times 1 \times 2 \mathrm{~mm})$ at the maximum of the electric and magnetic fields were investigated. For a series of 10 samples of PFN ceramics, the dielectric constant is $\varepsilon=59.8 \pm 8.2$. Similar measurements were conducted for samples of ferromanganite $\varepsilon=4.7 \pm 1.1, \mu=1.4 \pm 0.2$ and composite material $\varepsilon=36.7 \pm 5.1, \quad \mu=1.1 \pm 0.1$. The dielectric constant corresponds to a two-component statistical mixture and can be calculated using the formula:

$$
\varepsilon=A+\sqrt{A^{2}+\frac{\varepsilon_{1} \varepsilon_{2}}{2}}, A=\frac{1}{4}\left[\left(3 v_{1}-1\right) \varepsilon_{1}+\left(3 v_{2}-1\right) \varepsilon_{2}\right],
$$

where $v_{1}$ end $v_{2}$ are volume fractions of the composite material components.

In the frequency range of $8-40 \mathrm{GHz}$, the transmission and reflection coefficients were obtained for samples of the
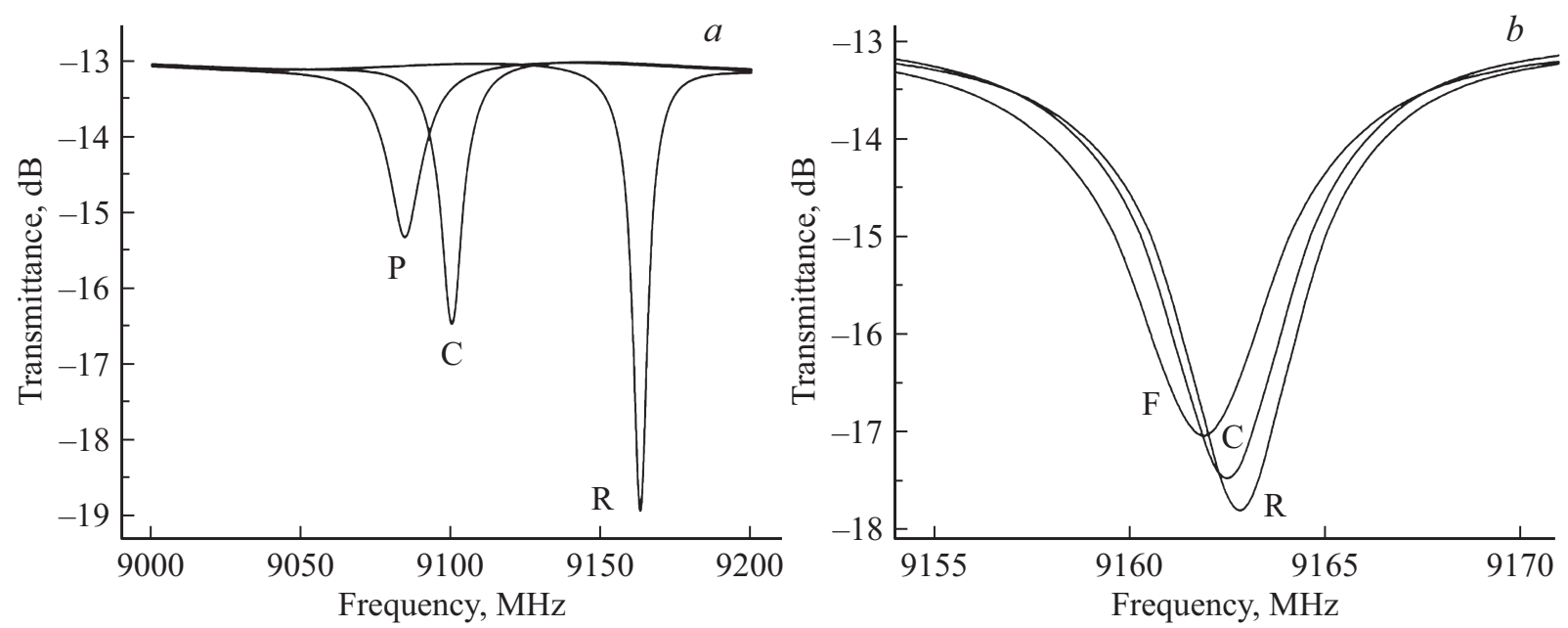

Figure 7. Resonance curves for empty resonator (R), PFN (P), CMF (F) samples and composite material (C) at the maximum of the $a$ ) electric and $b$ ) magnetic fields. 


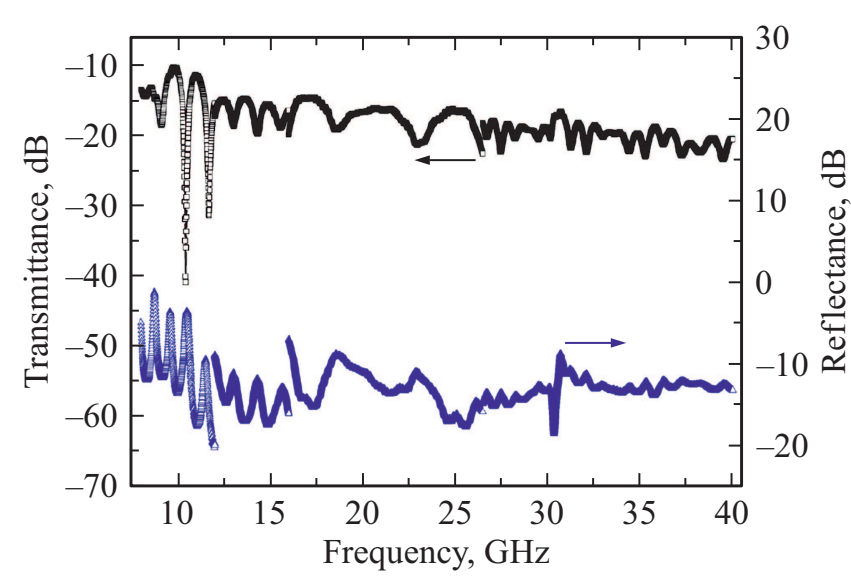

Figure 8. Transmittance and reflectance of a rectangular waveguide with a material sample of $0.5 \mathrm{PFN}+0.5 \mathrm{CMF}$.

composite material $10 \mathrm{~mm}$ long, $1 \mathrm{~mm}$ thick and height corresponding to the section of the $\mathrm{X}, \mathrm{Ku}, \mathrm{K}$, and $\mathrm{Ka}$ waveguides of the subbands (Fig. 8).

The sample under study appears to be a dielectric resonator in the measuring line. The resonances at frequencies $f=12.4$ and $12.6 \mathrm{GHz}$ result from the sample length, multiple of $\lambda / 2$. The reflection maxima in the rest of the range correlate well with the transmission minima. In addition, from the frequency dependences of transmission and reflection, it can be concluded that there is a small relative change in the material impedance in the investigated range.

\section{Conclusions}

When synthesized in an oxygen atmosphere, singlephase samples with a high degree of crystallinity can be obtained by two-stage annealing. In this case, the sintering temperature of the precursor and lead oxide should not exceed 1143 K. Studies of the obtained samples in the lowfrequency region of $20 \mathrm{~Hz} \leq f \leq 300 \mathrm{kHz}$ at various temperatures make it possible to determine the second-order phase transition temperature $T_{\mathrm{C}} \approx 370 \mathrm{~K}$. At temperatures below the Curie point $T_{\mathrm{C}}$, the PFN exhibits maximum dielectric losses at a frequency of $f \approx 1.5 \mathrm{kHz}$. High values of electrical conductivity in the composite material mask the maximum of dielectric constant corresponding to ferroelectric and phase transitions. The combination of ferroelectric and ferromagnetic phases in the composite material $0.5 \mathrm{PFN}+0.5 \mathrm{CMF}$ leads to the appearance of aggregated properties [2] (values of permittivity and magnetic permeability), as well as new properties and effects (change in the complex dielectric constant in the external magnetic field).

In the investigated composite material, the resonant nature of the magnetodielectric effect was revealed, which is due to its Maxwell-Wagner mechanism of occurrence. The experimental data obtained in this work indicate high values of lead ferroniobate ceramics dielectric constant in the frequency range $8 \leq f \leq 40 \mathrm{GHz}$.

\section{Conflicts of interests}

The authors declare that they have no conflict of interest.

\section{References}

[1] D.M. Evans, M. Alexe, A. Schilling, A. Kumar, D. Sanchez, N. Ortega, R.S. Katiyar, J.F. Scott, J.M. Gregg. Adv. Mater. 27, 39, 6068 (2015).

[2] N.A. Hill. J. Phys. Chem. 104, 29, 6694 (2000).

[3] O. Raymond. J. Appl. Phys. 97, 8, 084107 (2005).

[4] Y. Yang, J.-M. Liu, H.B. Huang, W.Q. Zou, P. Bao, Z.G. Liu. Phys. Rev. B 70, 13, 132101 (2004).

[5] A. Ashfaq. Proceed. SPIE 2780, Metal/Nonmetal Microsystems: Phys. Technol. Applications 106 (1996).

[6] G. Roussy, M. Felden. IEEE Trans. Microwave Theory. Techniques 14, 4, 171 (1966).

[7] A. Kania, E. Talik, M. Kruczek. Ferroelectrics 391, 1, 114 (2009).

[8] S. Singh, D. Pandey. Appl. Phys. Lett. 90, 24, 242915 (2007).

[9] A.V. Pavlenko, A.V. Turik, L.A. Reznichenko, L.A. Shilkina, G.M. Konstantinov. Phys. Solid State 53, 9, 1872 (2011).

Редактор Е.В. Толстякова 